\title{
Oinginal

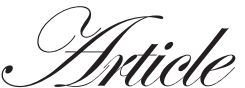 \\ A paradoxical role for an acute phase reactant in decompensated cirrhosis
}

\author{
George Peter, Paul Cheruvathoor George, Mashhood \\ Padincharepurathu Villyoth, Suthanu Bahuleyan, Nikhil Suraj, \\ Chethan Govindaraju, Shanid Abdul Sathar, Srijaya Sreesh, \\ Premalatha Narayanan, Kattoor Ramakrishnan Vinaya Kumar
}

ABSTRACT

Department of Medical

Background: This study aims to determine the predictive power of baseline C-reactive protein

Gastroenterology,

Government Medical College,

Thiruvananthapuram,

Kerala, India

Correspondence:

Dr George Peter

Email:georgepeter23@gmail.com

(CRP) value in cirrhotic patients with ascites, without overt infection, that might lead to spontaneous bacterial peritonitis (SBP)/ cellullitis

Methods: 152 consecutive cirrhotic patients with ascites, without overt infection were included in the study, after measuring the baseline CRP value. All patients were followed up for a duration of one year, or till development of SBP/ cellulitis.

Results: Baseline CRP was elevated in $76.8 \%$ of the patients. Development of infection was observed in $78(51.3 \%)$ patients. SBP was diagnosed in 54 patients, cellulitis was documented in 15 patients. 9 patients had simultaneous SBP and cellulitis Baseline CRP was $10.2 \pm 6.34 \mathrm{mg} /$ $\mathrm{dL}$ in the group who developed infection, it was $4.81 \pm 4.41 \mathrm{mg} / \mathrm{dL}$ in the group who did not develop infection ( $\mathrm{p}=0.002$ ). Baseline $\mathrm{CRP}>9.5 \mathrm{mg} / \mathrm{dL}$, serum albumin $<2.8 \mathrm{~g} / \mathrm{dL}$ and a previous history of infection were independent predictors of developing SBP/ cellulitis.

Conclusions: Along with low serum albumin and previous history of infection, CRP can be used as a predictive tool for early detection of infection, thus enabling to reduce the morbidity and mortality.

KEYWORDS: bacterial translocation, cellulitis, CRP, SBP, serum albumin

\section{Introduction}

The clinical course of decompensated cirrhosis is often studded with multiple complications which include bacterial infection, variceal bleeding, hepatocellular carcinoma, renal complications like hepatorenal syndrome, and hepaticencephalopathy. ${ }^{1}$ With advances in this field, clinicians are now able to cope with some of these complications like variceal bleeding (via interventional endotherapy), renal complication (via albumin and terlipressin regimes), whilst some others still remain unconquered. ${ }^{2,3}$ Bacterial infection ranks as the most important complication in this patient type. Thus early diagnosis and intervention in these cases is important. ${ }^{4}$

Spontaneous bacterial peritonitis and cellulitis are the most 
common infections encountered in a patient with decompensated cirrhosis. ${ }^{5,6}$ Various studies have probed the pathogenesis of these infections in this population and bacterial translocation has been implicated as a major causative factor. ${ }^{7,8}$ By advocating prophylactic antibiotics like norfloxacin in the susceptible population, it has been shown to decrease the disease burden. ${ }^{9}$ However the last few years have witnessed are emergence of these infectious complications, posing management challenges to the treating physicians. ${ }^{10,11}$

C-reactive protein (CRP) is an acute phase reactant, synthesized in the liver, as a response to systemic infection and inflammation, mediated by various cytokines like interleukin-6 (IL-6). ${ }^{12,13}$ It's clinical utility has been proved in the management of systemic infections and inflammatory disease states. ${ }^{14}$ Baseline CRP values are persistently elevated in patients with decompensated cirrhosis, even in the absence of infection. ${ }^{15}$ It has been shown that it is proportional to the magnitude of bacterial translocation and intestinal inflammation in these patients. ${ }^{16}$ However due to liver dysfunction, its clinical utility in acute infectious episodes is questionable, though there are studies supporting its role in guiding treatment course. ${ }^{17}$

In this context, this study was undertaken to study the benefit of monitoring elevated baseline CRP values in decompensated cirrhotic patients without overt infection. In the absence of overt infection, elevated baseline CRP might imply ongoing bacterial translocation, and hence indicate the susceptibility to potential SBP/cellulitis

The aim of this study was to determine the predictive power of CRP values in cirrhotic patients with ascites, without overt infection, with potential to develop future SBP/cellulitis.

\section{Methods}

This prospective observational study was conducted in a tertiary care center in South India, from November 2013 October 2014, after obtaining consent from the institutional research board. All consecutive cirrhotic patients with ascites, but without evidence of overt bacterial infection were enrolled into the study. The diagnosis of cirrhosis was based on a combination of clinical, biochemical, imaging features, and endoscopic evidence of esophageal or gastric varices, and histological features, where available. Blood samples and clinical data, including severity of cirrhosis were graded according to the Child-Pugh score and the MELD (Model for end stage liver disease) score, presence and grade of ascites, hepatic encephalopathy and recent episode of variceal bleeding, recorded at enrollment. Furthermore, presence of actual bacterial infection was actively sought on enrollment, including paracentes is and ascitic fluid study in all patients. Patients with mixed ascites were excluded. The diagnosis of infection was established by evaluation of clinical symptoms, appearance off ever and laboratory reports, including microbiological culture results (if available), and compatible findings by imaging techniques. Infections of skin and soft tissue (cellulitis), oral cavity, upper and lower respiratory tract (acute bronchitis, pneumonia), biliary tree (cholecystitis, cholangitis, liver abscess), intestinal tract (gastroenteritis), urinary tract (cystitis, pyelonephritis), osteomyelitis, and endocarditis were diagnosed based on conventional criteria. ${ }^{18}$ Inpatients with ascites, a diagnosis of spontaneous bacterial peritonitis was made if the ascetic fluid polymorpho nuclear cell (PMN) count was greater than $250 / \mathrm{mm}^{3}$, with or without positive culture, in the absence of an intra-abdominal source of infection. ${ }^{19}$ Clinical data, including age at onset, etiology, presence of esophageal varices and comorbidities, was collected retrospectively by thorough review of the patients' medical charts. Priorhi story of SBP orcellulitis, and details of antibiotic prophylaxis were documented The group of patients with out infection were enrolled into a prospective study to assess the incidence of SBP and cellullitis, after obtaining baseline CRP levels. Data were collected during the regular, follow-up visits. The follow-up period was for 12 months in those patients who showed no infectious episode, or until the date of appearance of infectious episode (SBP/cellullitis) in those patients with infection. The infections sought for during followup were SBP and cellullitis. Those patients who were lost to followup and those who died due to unrelated cause were excluded from the study.

Variables were expressed as mean \pm SD or median / inter quartile range, frequency and percentage, as appropriate, depending on their distribution. Differences between and within groups were assessed by unpaired or paired Student t-tests, Mann-Whitney U or Wilcox on tests, and Chi-squared test or ANOVA (analysis of variance) for repeated measures, as appropriate. Spearman's and Pearson's tests were used to determine correlations, as appropriate. Binary logistic regression was performed for those variables which were found to be significant during the univariate analysis. Receiver operating characteristic (ROC) curve analysis was applied to the independent prognostic factors, to calculate the area under the curve (AUROC), respective $95 \%$ confidence interval (CI) 
and the ideal cut-off value. Statistical analysis was performed using SPSS for Windows version 17.0. AP-value $<0.05$ was considered statistically significant.

\section{Results}

Of the 152 patients who were included in the final analysis of the study, 112(73.6\%) were male and 40(26.4\%) female. The median age was 58 (range 30-82) years. The etiology of cirrhosis was alcohol in 71 patients $(46.7 \%)$, hepatitis B virus (HBV) in $25(16.3 \%)$, non-alcoholic steatohepatitis (NASH) in $22(14.4 \%)$, alcohol+HBV in $12(7.9 \%)$, hepatitis C virus (HCV) in $12(7.9 \%)$, autoimmune hepatitis in 5(3.2\%), alcohol+HCV in $3(1.5 \%)$ and cryptogenic in $2(1.3 \%)$ patients. Child $\mathrm{C}$ patients accounted for $82.8 \%$ (126 patients), while $17.2 \%$ (26 patients) were categorized as ChildB. There were no Child-A patients in the study group. Ascitic fluid protein was $<1 \mathrm{~g} / \mathrm{dL}$ in $92(60.5 \%)$ patients.

Amongst the study subjects, 78 patients developed an infectious episode in the study period. Whilst SBP was diagnosed in 54 patients, cellulitis was documented in 15 patients. 9 patients had simultaneous SBP and cellulitis. Culture positivity rates were $20.3 \%$ (11 patients) in SBP, 26.6\% (4 patients) in cellulitis and $77.7 \%$ (7 patients) in combined infection. Mortality was $24.4 \%$ (18 patients) in this subgroup, while it was $5.4 \%$ (4 patients) in the 74 patients who did not develop an infectious complication in the study period, which was statistically significant $(\mathrm{p}<0.001)$.Culture positivity also had significant impact on mortality $(\mathrm{p}=0.005)$.

Baseline CRP was $10.2 \pm 6.34 \mathrm{mg} / \mathrm{dL}$ in the group who developed infection, it was $4.81 \pm 4.41 \mathrm{mg} / \mathrm{dL}$ in the group who did not develop infection ( $\mathrm{p}=0.002$ ). Baseline $\mathrm{CRP}$ was elevated in $76.8 \%$ of the patients $\quad \pm 6.310 .2 \pm 6.3$

Priorhi story of infection (SBP \pm cellulitis) was noted in 32 $(41.8 \%)$ patients in the infected group, whilst it was 16 (22.8\%) in the non-infected group $(\mathrm{p}<0.001)$. While $49(63.2 \%)$ patients in the infected group were on antibiotic prophylaxis, 54(74\%) patients in the non-infected group were on prophylactic antibiotics $(\mathrm{p}=0.089)$

Amongst the 78 patients who developed infection, 21
Table1: Clinical and laboratory parameters of patients with and without infection ( $\mathrm{SBP} \pm$ cellulitis)

\begin{tabular}{llll}
\hline & \multicolumn{2}{c}{ Infection (SBP/Cellulitis) } & P value \\
& Yes $(\mathbf{n = 7 8})$ & No $(\mathbf{n}=\mathbf{7 4})$ & \\
\hline Bilirubin $(\mathrm{mg} / \mathrm{dl})$ & $2.54 \pm 1.76$ & $1.15 \pm 1.78$ & 0.005 \\
AST(U/L) & $80(50-142)$ & $71(48-124)$ & 0.215 \\
ALT(U/L) & $49(40-94)$ & $45(31-84)$ & 0.891 \\
ALP(IU/L) & $111.57 \pm 55.90$ & $100.16 \pm 35.11$ & 0.420 \\
Albumin (g/dl) & $2.66 \pm .83$ & $2.96 \pm .64$ & $<0.001$ \\
INR & $1.87 \pm .96$ & $1.65 \pm .81$ & 0.085 \\
Creatinine (mg/dl) & $.86 \pm .47$ & $.79 \pm .25$ & 0.04 \\
Sodium (mEq/L) & $128.86 \pm 4.02$ & $129.53 \pm 5.20$ & 0.09 \\
Hemoglobin (g/dl) & $10.70 \pm 1.15$ & $11.58 \pm 1.70$ & 0.48 \\
Platelet (lakhs/mm $\left.{ }^{3}\right)$ & $1.41 \pm .69$ & $1.98 \pm .75$ & $<0.001$ \\
CRP(mg/dL) & $10.2 \pm 6.3$ & $4.81 \pm 4.41$ & 0.002 \\
Diabetes Mellitus & $19(25.5 \%)$ & $11(16.6 \%)$ & 0.017 \\
MELD score & $15.93 \pm 5.59$ & $14.55 \pm 5.87$ & $<0.001$ \\
Antibiotic prophylaxis $49(63.2 \%)$ & $54(74.0 \%)$ & 0.089 \\
Priorh/infection & $32(41.8 \%)$ & $16(22.2 \%)$ & $<0.001$ \\
\hline Values are expressed as
\end{tabular}

Values are expressed as mean $+S D$ or median (interquartile range), depending upon their distribution AST, aspartate aminotransferase; $A L T$, alanineamino transferase; ALP, alkaline phosphatase; INR, international normalized ratio; CRP, C-reactive protein; MELD, Model for End-stage Liver disease; h/o, history of

patients developed it in the first 4 months, whilst 14 developed it in the second four months, 26 developed in the third quarter and 17 in the last quarter $(\mathrm{p}=0.26)$. Nosocomial infection was documented in $15(19.2 \%)$ patients, and it had no correlation with the baseline $\mathrm{CRP}(\mathrm{p}=0.09)$. Table 1 summarises the baseline characteristics of patients who developed and did not develop an infectious complication.

On univariate analysis serum albumin, platelet count, baseline CRP, presence of diabetes mellitus, MELD score and previous history of infection were associated with development of infection. On binary logistic regression, baseline CRP value, serum albumin and previous history of infection emerged as in dependent predictors for development of infection in patients with decompensated cirrhosis. Table 2 represents the results of multivariate analysis with best cut-off values for the significant variables.

\section{Discussion}

This study was undertaken to identify the long term clinical

Table 2: Results of multivariate analysis with best cut-off values for significant variables to predict development of infection

\begin{tabular}{llll}
\hline Variable & Exp (B) (95\% CI) & P value & AUROC(95\%CI) \\
\hline CRP(mg/dL) & $19.5(1.9-193.8)$ & 0.017 & $0.890(0.772-1.000)$ \\
Albumin(g/dL) & $7.739(1.9-45.7)$ & 0.036 & $0.795(0.693-0.897)$ \\
Prior h/o infection & $7.4(1.1-83.7)$ & 0.040 & n.a. \\
\hline
\end{tabular}

AUROC, area under the receiver operating characteristic curve; $C R P, C$-reactive protein; h/o, history of; n.a., not applicable 
utility of CRP in patients with decompensated cirrhosis. CRP is an acute phase reactant and has a role in guiding management course in patients with systemic inflammation and infection. However, in a patient with hepatic dysfunction, CRP does not elevate appropriately and hence confounds clinical judgment. The long term impact of an elevated acute phase reactant has never been studied before. Even though the half life of CRP is only 18.2 hours, its chronic sustained elevation has prognostic significance in this patient population.

Bacterial translocation, the passage of bacteria or their products from the gut into the circulation, is a major mechanism in the development of bacterial infections in cirrhosis, especially SBP and cellulitis. With emerging data supporting Gram negative bacilli as causative organisms for cellulitis (similar to $\mathrm{SBP}$ ) bacterial translocation emerges as the main pathogenetic mechanism behind cellulitis in cirrhosis. Sustained chronic bacterial translocation without overt inflectional so has remarkable impact on the pathogenetic processes of the disease (elevated proinflammatory cytokine levels and decreased vascular resistance) ${ }^{18}$ Identification of patients at risk for developing future infections is of utmost importance as far as management of decompensated cirrhotic patients are concerned. ${ }^{20}$

Elevated baseline CRP $>9.5 \mathrm{mg} / \mathrm{dL}$ will serve as an easily measurable prognostic tool for the treating physician, enabling more efficient followup for that respective patient. Whenever, the clinical course of decompensated cirrhosis is complicated with acute events like alcoholic hepatitis, pancreatitis, myocardial injury, the coexistence of infection has significant impact on the management and outcome. However, CRP fails to aid the clinician in making a distinction in this setting, which has led to the decline of its use by hepatologists. This study has reignited the clinical utility of CRP in an entirely different context, which is a paradox, as far as this peculiar setting of decompensated cirrhosis and bacterial translocation is concerned.

Our study draws attention to certain negative findings concerning the same, e.g. it has no role in predicting the onset of a nosocomial infection. A higher magnitude of baseline CRP does not imply potential infection.

The last two decades has witnessed arise in the incidence of infection even in the setting of antibiotic prophylaxis, which has raised concern. Our study has shown a similar paradox, with a greater proportion of patients on antibiotic prophylaxis developing an infectious complication. This emphasizes the need for revising our antibiotic prophylaxis guidelines and utilizing more gut sterilizing antibiotics to combat bacterial translocation and efforts to address the issue of changing intestinal microbiota.

Past history of infection, emerging as an independent predictor of developing infection as per our study, may be a direct indicator of persistent bacterial translocation, which has remained unattended to after the initial episode. Low serum albumin is an established risk factor for bacterial infection in these patients, further validated in our study.

There are limitations in our study. The CRP value was measured at baseline, and any alterations during the study period were not accounted for, as it would have interfered with the clinical utility of the baseline CRP. However, an elevated CRP at baseline was reconfirmed with a second value be fore enrollment. Secondly, our study population predominantly comprised Child C patients, with no Child A subjects, hence our results cannot be generalized to the entire spectrum of cirrhotic patients. Inclusion of only cirrhotic patients with ascites has led to this disparity. Thirdly, other common infections like urinary tract infections, biliary infections, respiratory tract infections were not included, primarily because the role of bacterial translocation in causing infections at these sites remains controversial. Finally, the role of other inflammatory markers like procalcitonin has not been included, due to paucity of data claiming its significance in bacterial translocation.

To conclude, though CRP's predictive power for infection has a declining role in the setting of decompensated and advanced cirrhosis; it has found a new, rather unexpected role to playing predicting the development of a SBP or cellulitis in a decompensated cirrhotic patient without overt infection. Its new found clinical utility, after validation studies, might go along way in helping the physician in the management of decompensated liver disease, where infections continue to remain a growing menace.

\section{Abbreviations}

AST, aspartate aminotransferase; ALT, alanine aminotransferase; ALP, alkaline phosphatase; INR, international normalized ratio; CRP, C-reactive protein; MELD, Model for End-stage Liver disease; h/o, history of; ANOVA, analysis of variance; AUROC, area under the receiver operating characteristic curve; ROC, receiver operating characteristic curve; $\mathrm{CI}$, confidence interval; SD, standard deviation; SBP, spontaneous bacterial peritonitis; IL- 
6, interleukin-6; $\mathrm{HCV}$, hepatitis $\mathrm{C}$ virus; $\mathrm{HBV}$, hepatitis B virus; $\mathrm{NASH}$, non alcoholic steatohepatitis; PMN, polymorpho nuclear

\section{Acknowledgement}

The authors have no conflicts of interest to disclose

\section{References}

1. Dong MH, Saab S. Complications of cirrhosis. Dis Mon.2008;54:445-56.

2. Imperiale $\mathrm{TF}^{1}$, Chalasani N.Ameta-analysis of endoscopic variceal ligation for primary prophylaxis of esophageal variceal bleeding. Hepatology. 2001;33:802-7.

3. Rajekar H, Chawla Y. Terlipressin in hepatorenal syndrome: Evidence for present indications. J Gastroenterol Hepatol. 2011;26:109-14.

4. Pleguezuelo $\mathbf{M}^{1}$, Benitez JM, Jurado J, Montero JL, De la Mata M. Diagnosis and management of bacterial infections in decompensated cirrhosis. World J Hepatol. 2013;5:16-25.

5. BorzioM, Salerno F, Piantoni L, Cazzaniga M, Angeli P, Bissoli F, et al. Bacterial infectionin patients with advanced cirrhosis: a multicentre prospective study. Dig Liver Dis. 2001;33:41-8.

6. Swartz ML, Pasternack MS. Cellulitis and superficial infections. Prin Pract Inf Dis. 1999;88:34-7

7. J M Llovet, R Bartolí, R Planas, E Cabré, M Jimenez, A Urban, et al. Bacterial translocation in cirrhotic rats. Its role in the development of spontaneous bacterial peritonitis. Gut. 1994;35:1648-52.

8. JM Llovet, R Bartoli, R Planas et al. Bacterial translocation in cirrhotic rats. Its role in the development of spontaneous bacterial peritonitis. Gut 1994;35:1648-65.

9. 9 Tarsila CR Ribeiro, Julio MF Chebli, Mario Kondo, Pedro Duarte Gaburri, Liliana Andrade Chebli, Ana Cristina Amaral Feldner. Spontaneous bacterial peritonitis: How to deal with this life-threatening cirrhosis complication? Ther Clin Risk Manag. 2008;4:919-25.

10. Novella M, Solà R, Soriano G, Andreu M, Gana J, Ortiz J, et al.
Continuous Versus Inpatient Prophylaxis of the First Episode of Spontaneous Bacterial Peritonitis With Norfloxacin. Hepatology. 1997;25:532-6.

11. Tandon P, Delisle A, Topal JE, Garcia-Tsao G. High Prevalence of Antibiotic-Resistant Bacterial Infections Among Patients With Cirrhosis at a US Liver Center. Clin Gastro enterol Hepatol. 2012;10:1291-8

12. Steel DM, White head AS. The major acute phase reactants: Creactive protein, serum amyloid $\mathrm{P}$ component and serum amyloid A protein. Immunology today. 1994;15:81-8.

13. Blüher M., Fasshauer M, Tonjes A, Kratzsch J, Schon MR, Paschke R. Association of interleukin-6, C-reactive protein, interleukin-10 and adiponectin plasma concentrations with measures of obesity, insulin sensitivity and glucose metabolism. Exp Clin Endocrinol Diabetes. 2005;113:534-7.

14. Xu SY, Pauksen K, Venge P. Serum measurements of human neutrophillipocalin (HNL) discriminate between acute bacterial and viral infections. Scand J Clin Lab Invest. 1995;55:125-31.

15. ChoY, Park SY, Lee JH, Lee DH, Lee M, Yoo JJ, et al. Highsensitivity C-reactive Protein Levelisan Independent Predictor of Poor Prognosis in Cirrhotic Patients With Spontaneous Bacterial Peritonitis. J Clin Gastroenterol. 2014;48:444-9.

16. Çevikel MH, Osqun H, Boylu S, Demirkiran AE, Aydin N, Sari $\mathrm{C}$, et al. $\mathrm{C}$ reactive protein may be a marker of bacterial translocation in experimental intestinal obstruction. ANZJS. 2004;74:900-4.

17. Tsiakalos A, Karatzaferis A, Ziakas P, Hatzis G. Acute phase proteins as indicators of bacterial infection in patients with cirrhosis. Liver International. 2009;29:1538-42.

18. Papp M, Vitalis Z, Altorjay I, Tornai I, Udvardy M, Harsfalvi J, et al. Acute phase proteins in the diagnosis and prediction of cirrhosis associated bacterial infections. Liver International. 2012;32:603-11.

19. European Association for the Study of the Liver EASL clinical practice guidelines on the management of ascites, spontaneous bacterial peritonitis, and hepatorenal syndrome in cirrhosis. Journal of Hepatology. 2010;53:397-417.

20. Giulia P, Agarwal B, Burroughs AK. C-reactive protein and bacterial infection in cirrhosis. Annals of Gastroenterology: Quarterly Publication of the Hellenic Society of Gastroenterology. 2014;27:113. 